Research, Society and Development, v. 9, n. 5, e15952575, 2020

(CC BY 4.0) | ISSN 2525-3409 | DOI: http://dx.doi.org/10.33448/rsd-v9i5.2575

\title{
O processo de avaliação psicológica: estudo de caso
}

The process of psychological assessment: case study

El proceso de evaluación psicológica: estudio de caso

Recebido: 16/01/2020 | Revisado: 10/02/2020 | Aceito: 03/03/2020 | Publicado: 24/03/2020

Gabriela Maffini

ORCID: https://orcid.org/0000-0001-9130-4699

Universidade Franciscana, Brasil

E-mail: g_maffini@hotmail.com

Paula Argemi Cassel

Mestre em Psicologia pela Pontifícia Católica do Rio Grande do Sul , Brasil

ORCID: https://orcid.org/0000-0003-2556-4067

E-mail: Paula.acassel@hotmail.com

\section{Resumo}

O artigo tem por objetivo apresentar e descrever os resultados de um estudo de caso desenvolvido a partir de um psicodiagnóstico clínico realizado com uma pessoa do sexo feminino, de faixa etária adulta, em contexto de clínica-escola, localizada em uma Universidade privada do interior do Rio Grande do Sul - RS. Para que os objetivos fossem atingidos, realizaram-se o total de oito sessões psicológicas, onde triagem, anamnese, testagem e a devolução ocorreram. Como instrumentos de testagem, para apontar as forças e fraquezas da paciente, utilizou-se da Bateria Fatorial de Personalidade - BFP, de entrevistas clínicas e observação clínica. A metodologia adotada foi um estudo de caso único e sistemático, com caráter exploratório. Como resultado, a partir do psicodiagnóstico, foi possível levantar informações sobre a história de vida da paciente, compreender e avaliar a personalidade da mesma e a influência significativa da família nos processos de tomada de decisão da examinanda. Tal processo, permitiu a realização de um encaminhamento e de indicação terapêutica para que as necessidades da examinanda fossem atendidas de forma adequada.

Palavras-chave: Psicodiagnóstico; Estudo de caso; Clínica-Escola. 


\section{Abstract}

This article aims to present and describe the results of a case study developed from a clinical psychodiagnosis performed with a women of adult age, in the context of a school-clinic, located in a private university in the interior of Rio Grande do Sul - RS. In order to achieve the objectives, a total of eight psychological sessions were perfomed, where screening, anamnesis, testing and oral return happened. As a testing instrument, to point out the patient's strengths and weaknesses, it was used the Personality Factorial Battery - BFP, clinical interviews and clinical observation. The methodology adopted was a unique and systematic exploratory case study. As a result, from the psychodiagnosis, it was possible to gather information about the patient's life history, understand and evaluate her personality and the significant influence of the family on the decision-making processes of the examiner. This process allowed for therapeutic indication so that the patient's needs were adequately met.

Keywords: Psychodiagnosis; Case Study; School Clinic

\section{Resumen}

El artículo tiene como objetivo presentar y describir los resultados de un estudio de caso desarrollado a partir de un psicodiagnóstico clínico realizado con una mujer, en edad adulta, en el contexto de una escula-clínica, ubicada en una universidad privada en el interior del estado Rio Grande do Sul - RS. Para alcanzar los objetivos, se llevaron a cabo un total de ochou sesiones psicológicas, donde se realizaron exámenes de detección, anamnesis, evaluacion psicológica y retorno oral. Como instrumento, para señalar las fortalezas y debilidades del paciente, utilizamos la Batería Factorial de Personalidad - BFP, entrevistas clínicas y observación clínica. La metodologia adoptada fue un estudio de caso exploratorio único y sistemático.. Como resultado, a partir del psicodiagnóstico, fue posible recopilar inforción sobre la historia de vida de la paciente, compreender y evaluar sua personalidad y la influencia significativa de la familia en los procesos de toma de decisiones de la paciente. Este proceso permitió la derivación y la indicación terapéutica para satisfacer adecuadamente que las necesidades de la paciente.

Palabras clave: Psicodiagnóstico; Estudio de caso; Clínica escolar. 


\section{Introdução}

A avaliação psicológica clínica com fins diagnósticos é considerada como uma prática cada vez mais comum no Brasil, sendo evidenciado ao passar dos anos, um aumento recorrente pela procura de atendimentos com a finalidade do psicodiagnóstico (Cunha \& Benetti, 2009). Com base na necessidade de ressaltar a importância do processo de avaliação, o presente estudo tem como objetivo descrever os passos de um psicodiagnóstico e apresentar, a partir da descrição de um caso clínico, os procedimentos que compuseram o processo de avaliação. $\mathrm{O}$ atendimento realizado refere-se à prática de um estágio profissional vinculado ao serviço de clínica-escola de Psicologia de uma Universidade privada do Rio Grande do Sul.

A avaliação psicológica utiliza um conjunto de técnicas para sua realização, estendendo-se para além de apenas o uso de testes psicológicos para obtenção de um resultado, recorrendo a outros instrumentos como entrevistas clínicas (estruturadas e semiestruturadas) e observações do paciente. Quando se enunciado o conceito de avaliação psicológica, a falta de conhecimento sobre a área acaba por associar este processo a apenas ao uso de testes psicológicos. Para diferenciar estes conceitos o Conselho Federal de Psicologia CFP, elaborou uma cartilha no ano de 2013, apontando que o conceito de avaliação psicológica é um processo que envolve a integração de diversas fontes de informações, entre elas, testes, entrevistas e observações, enquanto que a testagem envolve exclusivamente como fonte de informação, os testes psicológicos (CFP, 2013).

A lei federal número 4.119 de 27 de agosto de 1962, dispõe sobre a formação em Psicologia e regulamenta a profissão no Brasil, definindo que a prática do diagnóstico psicológico, bem como a realização do psicodiagnóstico, é atribuição exclusiva do psicólogo (Brasil, 1962). Através deste processo, se faz possível compreender e levantar dados sobre o examinando e sua família, com o objetivo de investigar aspectos da personalidade, aspectos afetivos, sociais e cognitivos, bem como abordar possíveis sintomas, questões neuropsicológicas, questões de desenvolvimento, entre outros, para que seja possível fazer um encaminhamento que vá de acordo com as necessidades do examinando (Rigoni \& Sá, 2016).

Ressalta-se que este procedimento é delimitado no tempo, com número definido de encontros, o qual procura descrever as forças e fraquezas do funcionamento psicológico do indivíduo. Apesar de haver a delimitação no tempo de duração para que o psicodiagnóstico seja realizado, o processo não se delimita apenas ao individual pois pode envolver familiares e pessoas que cercam o examinando, a fim de obter uma maior compreensão do cotidiano do mesmo (Cunha, 2000). Para que esta compreensão ocorra, é essencial a presença de confiança 
e empatia na relação entre terapeuta-paciente, para que o mesmo sinta-se à vontade para falar sobre suas demandas, sem nenhum tipo de restrição ou receio, havendo também grande importância da existência da confiança para que este sinta-se a vontade que seus familiares/ pessoas, quando surgido necessidade, participem do processo. Do profissional de Psicologia, espera-se que não somente a escuta atenta do relato verbal seja feita, espera-se que também a escuta não verbal do comportamento seja realizada (Mônego, 2016).

Ainda que a comunicação verbal seja importante para o processo de avaliação psicológica, deve-se dar atenção para o que ocorre durante a sessão de forma não-verbal, visto que gestos, comportamentos, feições, são aspectos fundamentais para a compreensão sobre o sujeito ir além do que se é ouvido. Determinando-se a relevância do uso de ferramentas clínicas dentro do processo de avaliação psicológica irem além de entrevistas e uso de testes psicológicos, este artigo teve como intuito apresentar e descrever o resultado de um psicodiagnóstico clínico realizado com uma examinanda do sexo feminino, de faixa etária adulta, em contexto de serviço-escola.

\section{Triagem}

A primeira etapa do atendimento realizado nos serviços-escolas consiste na triagem, pois a partir desta que se torna definido a necessidade de direcionar mais especificamente para o processo de avaliação psicológica. A triagem estende-se para além de apenas uma única entrevista, envolvendo mais de um contato com objetivo de coleta de informações e dados do paciente, para que assim, seja possível estabelecer um curso de tratamento futuro. As configurações das entrevistas de triagem são semiestruturadas, sendo assim, o entrevistador tem clareza de seus objetivos e a partir do relato do examinando, este pode ir aprofundando questões que percebe que possuem maior importância pois não encontra-se preso em um roteiro rígido (Marques, 2005).

Para que o examinando saiba que o ambiente terapêutico é um local seguro para que seus problemas sejam relatados, é importante que ocorra um estabelecimento de um vínculo seguro e consistente logo nas sessões iniciais. A falta de confiança impede que o examinando inicie o seu relato por não conhecer o ambiente ou por se sentir inseguro de desabafar com o terapeuta que até então, é desconhecido para si. Destaca-se, assim, a importância do terapeuta dar início ao processo e indo além de apenas questionar, incentivando-o, para começar a relatar a sua queixa. $\mathrm{O}$ entrevistador pode utilizar de diversos recursos para obter maiores informações, como as intervenções através de perguntas, comentários, esclarecimentos e explicações, não sendo estes recursos utilizados somente para obter um maior entendimento 
da situação da pessoa mas para também proporcionar uma tomada de consciência sobre seus problemas (Cordioli, 1998).

O processo de triagem não envolve apenas um único encontro com o paciente, visto que, este não seria suficiente para coleta de todos os aspectos relevantes frente a demanda que o mesmo traz. Ainda, para que este processo ocorra de forma fluida, destaca-se a necessidade do psicoterapeuta não se prender a um roteiro rígido, estruturado, pois isso acaba por delimitar o aprofundamento em possíveis questões relevantes para o processo de avaliação e a necessidade de ocorrer o estabelecimento de vínculo entre terapeuta-paciente, para que este sinta-se à vontade no ambiente terapêutico, sendo livre para relatar o que deseja, sem receio de possíveis julgamentos.

\section{Anamnese}

A anamnese é um tipo de entrevista realizada para investigar a história do examinando, ou seja, os aspectos de sua vida considerados relevantes para a demanda trazida (Silva \& Bandeira, 2016). A realização da anamnese auxilia a trazer consciência os fatos relacionados a queixa e história do examinando, buscando a relação entre os aspectos de vida do sujeito e o problema apresentado (Cunha, 2000). Este processo é dependente da faixa etária do sujeito, pois com crianças é direcionada para os pais ou responsáveis, enquanto que na faixa etária do adolescente, pode ser feita com os pais e complementadas pelo adolescente, enquanto que no caso de adultos e idosos é realizado com os próprios, salvo em casos de psicopatologias severas (Silva \& Bandeira, 2016).

Possui caráter investigativo e prioriza o levantamento de informações cronologicamente organizadas que guiam a tomada de decisão sobre como prosseguir com o processo avaliativo (Cunha, 2000). A entrevista não segue a mesma estrutura para todos os examinandos, entretanto, certos aspectos sempre se fazem presentes na investigação independente da idade, como configuração e as relações familiares, características do avaliando e questões referentes a queixa. Para que esta seja feita de forma correta, envolve a necessidade de ter conhecimentos de áreas diversas, pois o conhecimento aprofundado auxilia o psicólogo a conduzir o relato e encontrar detalhes da queixa. Silva \& Bandeira (2016) consideram que a entrevista é um recurso essencial para o conhecimento do avaliando e para o adequado direcionamento do psicodiagnóstico.

O processo de anamnese foca em aspectos do desenvolvimento do sujeito, sendo uma fase de investigação referente a queixa e a história do examinando. Este processo depende da faixa etária do examinando, visto que, torna-se impossível para que um examinando da faixa 
etária infantil saiba sobre aspectos relevantes a gestação e seu desenvolvimento inicial. Ainda com adolescentes, este processo pode incluir os pais e ter participação do examinado, visto que, os pais podem não saber sobre aspectos relevantes do cotidiano do adolescente e este não saber sobre aspectos relevantes da sua infância. Já com adultos, este processo é exclusivamente realizado com os mesmos, salvo exceções.

\section{Observação clínica}

A observação ocupa um lugar significativo como método clínico e de pesquisa, envolvendo a ação de olhar com atenção os fenômenos para descrever, estudar e compreender aspectos intrínsecos. Dentro do campo psicológico, surge-se o objetivo de capturar aquilo que não é exclusivo a audição ou apenas do olhar destreinado, sendo necessária a atenção focada no paciente para compreensão dos comportamentos e sentimentos provenientes dos atendidos (Arpini, et. al, 2018).

Como meio de coleta de dados, aponta-se para a observação clínica como um dos instrumentos mais eficazes, pois a partir do olhar treinado do profissional, este reconhece aspectos que se a atenção fosse apenas direcionada a demanda verbal, os aspectos não iriam ter a sua necessária relevância. O psicólogo consegue identificar os recursos disponíveis dentro do ambiente do examinando e a partir disto, intervir com estratégias, visando a promoção de saúde do atendido (Britto, Oliveira, \& Sousa, 2003). Ainda que se tenha a ideia que o processo de avaliação psicológica seja exclusivamente o uso de testes, uma das técnicas fundamentais para este processo é a observação clínica para identificação de comportamentos, sentimentos despertados nos atendidos, como sensação de desconforto ou comportamento de esquiva, auxiliando no manejo do profissional com o examinando.

\section{Entrevista devolutiva}

A entrevista devolutiva é o momento onde ocorre a devolução oral das informações que compuseram o processo do psicodiagnóstico, qual deve englobar, os momentos vivenciados durante as etapas anteriores e qual deve produzir a integração de todos estes momento e a partir disto, conduzir um fechamento e abrir portas para novos direcionamentos. A devolução encerra um processo bastante complexo, responsável por ratificar a importância da realização do psicodiagnóstico e por produzir efeitos nos sujeitos que o vivenciaram (Albornoz, 2016).

Conforme o autor supracitado, é necessário que o profissional possa nomear e esclarecer ao avaliando, o sentido dos sintomas, localizando-os dentro do contexto 
apreendido, bem como sua importância e utilidade. Para que o processo seja finalizado, constata-se que é um momento importante para que sejam retomados aspectos da avaliação como a queixa inicial, as entrevistas pra conduzir um encerramento do processo.

\section{Laudo}

O laudo psicológico é um documento técnico, de natureza descritiva e demonstrativa, que expõe dados e argumenta sobre o que foi examinado, tendo como função esclarecer questões produzir conhecimento técnico especializado. $\mathrm{O}$ laudo responde questões do tipo "o que", "o quanto', “como", "porque", "para que" e "quando" e por isso costuma ser um processo bastante detalhado e a partir deste, o profissional consegue estabelecer o curso de tratamento a vir a ser seguido. (Hutz, et. al, 2016)

Além de ser importante para o profissional de Psicologia e para o examinando/familiares, o psicodiagnóstico preza pela interdisciplinaridade, ou seja, a troca de conhecimento entre os profissionais de outra área. O laudo é uma peça escrita quais os profissionais expõem suas observações e conclusões a que chegaram, sendo esta a última etapa do psicodiagnóstico, consistindo na devolução dos resultados e objetivos estabelecidos durante o processo. Ademais, a finalidade do laudo está associada diretamente a qualidade da intervenção (Guzzo \& Pasquali, 2001). O laudo é um instrumento qual permite que os aspectos avaliados no psicodiagnóstico, estejam transcritos em um documento técnico e profissional.

\section{Metodologia}

Este trabalho trata-se de um estudo de caso único, pois possui a intenção de investigar uma realidade e a compreender de forma integral, com o objetivo do desenvolvimento de conhecimento científico (Coimbra \& Martins, 2013), além de ser caracterizado por ser uma pesquisa qualitativa, definida por Gil (2002), como uma pesquisa-ação, onde a obtenção de dados e interpretação destes são os focos principais. Desenvolveu-se este estudo de caso, a partir de um processo de avaliação psicológica realizado no laboratório de práticas em Psicologia de uma Universidade privada, localizada no interior do Rio Grande do Sul - RS, com uma examinanda do sexo feminino, de idade adulta, tendo esta 44 anos. A examinanda assinou o Termo de Consentimento Livre e Esclarecido (TCLE), autorizando a realização de registros em prontuários e de que fossem relatadas em supervisões, os acontecimentos dos atendimentos. 
O período destinado à realização do psicodiagnóstico foram de aproximadamente três meses e meio, onde foram realizados, no total, oito encontros, onde triagem, anamnese, testagem, entrevista devolutiva e construção do laudo psicológico ocorreram. Apesar do processo de psicodiagnóstico ter iniciado no final de agosto do ano de 2018 , feriados e faltas da examinanda, ocasionaram o término do processo ser no final de novembro do mesmo ano. O teste psicológico utilizado foi a Bateria de Personalidade Fatorial - BFP (Nunes; Hutz; \& Nunes, 2010) para que aspectos da personalidade fossem destacados. A Bateria Fatorial de Personalidade (BFP), baseada no modelo dos Cinco Grandes Fatores (CGF) é um instrumento psicológico construído para a avaliação da personalidade que inclui as dimensões: extroversão, socialização, realização, neuroticismo e abertura a experiências (Nunes; Hutz; \& Nunes, 2017). Para que o processo de avaliação psicológica fosse o mais completo, utilizou-se de ferramentas além da observação clínica e de entrevistas semiestruturadas, incluindo-se o teste psicológico.

\section{Resultados e discussões}

A examinanda foi encaminhada pelo serviço de residência multiprofissional de reabilitação da mesma Universidade privada, visto que possuía ansiedade e uma condição física que a impossibilitava determinadas ações cotidianas, delimitando-a fisicamente e psicologicamente, possuindo o diagnóstico de distrofia muscular de cinturas. A partir das sessões de triagem, a examinanda trouxe que o motivo da procura de atendimento psicológico estava associado com a queixa de que necessitava encontrar um espaço para desabafar sobre problemas provenientes de seu cotidiano e instituição familiar que a cercava.

Vanessa (nome fictício), possuía 44 anos no momento de realização dos atendimentos, sendo casada com o seu primeiro namorado desde os 23 anos, tendo uma filha, de 21 anos, como fruto do casamento. Mora com o marido, filha e sua prima, cujo é considerada como fonte de estresse constante para a família, pois ao mesmo tempo que a prima age de forma inadequada, com explosões de raiva, a examinanda e a família dependem dela financeiramente e para que a mesma auxilie Vanessa em tarefas básicas como ir ao banheiro e levantar da cama.

Durante o questionamento da estagiária sobre a sua árvore genealógica, a examinanda relatou que a sua história familiar era bastante complicada, tendo perdido a mãe para o suicídio quando havia um ano de idade. Apesar da perda da mãe ter acontecido quando havia um ano, a examinanda morava com a tia desde os seus 2 meses de idade, que é quem 
considera como figura materna, evidenciando-se nos atendimentos pois quando utilizava o termo "mãe", era sobre a tia quem referia-se. Já a relação com o seu pai, desde a infância até sua vida adulta é bastante conturbada por considerar como o motivo principal pela perda de sua mãe biológica, as constantes traições matrimoniais.

Considerava sua adolescência como rígida devido aos inúmeros limites impostos pela sua tia, de comportamento e locais que poderia frequentar. Morou a vida toda com a sua prima, sendo assim, percebia-se que a existência de uma dificuldade de estabelecer limites com a prima pela existência de uma história prévia e sentimento de culpa diante a possibilidade de ser considerada como ingrata pelos demais familiares que auxiliaram na sua criação. O contato com o pai durante a adolescência foi extremamente limitado, pois mesmo sua tia sendo irmã do seu pai, ela o culpava pela perda súbita da mãe de Vanessa e sendo assim, a tia impedia que este se fizesse presente em diversas ocasiões. Ao chegar na maioridade, perdeu a tia subitamente para um acidente vascular cerebral hemorrágico e apenas a partir deste momento que ocorreu a reaproximação com o pai, entretanto, a examinanda apontou que existiam diversas ressalvas e dificuldade de estabelecer um vínculo.

O diagnóstico da doença ocorreu durante seus anos iniciais de casada, destacando que o marido sempre esteve presente nos episódios mais difíceis de sua vida, além de que, aponta que sua doença influenciou na escolha do curso de graduação posterior do marido, pois este buscou adquirir conhecimento para auxiliar a mesma. Durante a sua vida adulta, passou por diversas hospitalizações devido a recorrência de problemas respiratórios, sendo está uma comorbidade do seu diagnóstico de distrofia. Em decorrência das internações, Vanessa desenvolveu uma fobia diante de ambientes hospitalares, tendo uma ansiedade constante em pensar na possibilidade de ser internada de novo, havendo um comportamento extremamente evitativo em relação a ir ao médico.

Em relação ao primeiro atendimento, houve uma surpresa em relação a quanto a examinanda conseguiu relatar para a estagiária, abordando diversos aspectos considerados delicados pela própria, apontando que nunca havia conseguido contar para ninguém fora a estagiária. Evidenciou-se assim, a necessidade imediata desta relatar sobre seus problemas de saúde qual considera limitantes e sobre a sua instituição familiar. De acordo com Beck (2013), é essencial a presença de confiança e rapport com o paciente desde o primeiro contato para que consiga sentir-se confortável em relatar seus sentimentos e pensamentos, dando início ao processo cuja aliança terapêutica é fundamental para que exista continuidade.

Nas sessões seguintes, a examinanda demonstrou bastante desagrado em relação a sua rotina pela dependência com seus familiares para se movimentar ou para que tarefas rotineiras 
em sua casa fossem realizadas. Ademais, a principal dificuldade nomeada por Vanessa, era relatar o desejo que a prima se mudasse de sua casa pois a convivência era complicada, sempre nomeando um sentimento de culpa associado ao seu desejo. Ela constantemente mudava de assunto quando o tópico de sua infância conturbada aparecia, percebia-se que a mesma utilizava de mecanismos de defesa evitativos. Os mecanismos de defesa compõem uma das formas do sujeito se afastar da ansiedade e evitar que aspectos dolorosos para o mesmo sejam discutidos, entretanto, destaca-se que nem sempre é uma ação consciente (Castilho, 2002).

No sexto atendimento, aplicou-se a Bateria Fatorial de Personalidade - BFP para que aspectos da personalidade fossem avaliados. A personalidade se refere as características dos indivíduos, levando em consideração a forma como o sujeito se comporta, pensa e sente suas emoções, sendo única e diferencial dos demais (Trentini, et. al, 2009). Os resultados do BFP foram bastante condizentes com o que a examinanda demonstrava nos atendimentos, existindo uma alta tendência em possuir dificuldade de estabelecer limites, preocupação com as necessidades dos outros em detrimento da sua vontade, dificuldade de confiar nas pessoas, sendo justificado pelos acontecimentos familiares, além de que possui uma tendência a ver o futuro de forma negativa. Outros aspectos evidenciados de Vanessa, é que a mesma é bastante dedicada e procura envolver-se nas tarefas propostas tanto em atendimento psicológico quanto fora da clínica, além de apresentar alta pontuação nos itens de extroversão e socialização.

No sétimo atendimento, houve devolução parcial do que havia sido conversado em atendimento e dos resultados da testagem. A devolução parcial é considerada como o início do desfecho do momento da devolução de informações (Albornoz, 2016). Este encontro, de forma sintetizada, falou previamente sobre aspectos considerados relevantes para auxiliar a examinanda a finalizar o processo do psicodiagnóstico. No último encontro, realizou-se a devolução dos aspectos evidenciados durante o atendimento, sendo apontado para Vanessa suas principais potencialidades, de comprometimento com o atendimento e com objetivos impostos para si e dificuldades, como estabelecer limites dentro de sua própria casa e o receio constante do que podem vir a pensar de suas ações. Ademais, se deu o encaminhamento para a examinanda, que seria indicada para psicoterapia de orientação cognitiva-comportamental, apontada como extremamente eficaz para manejo de sentimentos e emoções provenientes de eventos traumáticos e perdas não vivenciadas (Habigzang, et. al, 2009). A terapia cognitivacomportamental visa a resolução de problemas, a colaboração entre terapeuta-paciente, qual 
Research, Society and Development, v. 9, n. 5, e15952575, 2020

(CC BY 4.0) | ISSN 2525-3409 | DOI: http://dx.doi.org/10.33448/rsd-v9i5.2575

juntos atuam como uma equipe e tem por objetivo auxiliar o paciente a ser seu próprio terapeuta, aprendendo habilidades especificas para serem usadas durante a vida do paciente.

\section{Considerações finais}

O psicodiagnóstico é um processo qual visa a descrição e compreensão das forças e fraquezas do funcionamento psicológico do examinando, sendo este processo delimitado no tempo, com um número de encontros definidos de forma aproximada. Considerando os resultados encontrados, percebe-se que a examinanda avaliada seria indicada para atendimento psicoterápico, sendo a abordagem sugerida com o enfoque na terapia cognitivacomportamental, pois esta, a partir de uma avaliação realista e a modificação do pensamento, proporciona uma mudança comportamental e também emocional necessária para a mesma.

Com o devido acompanhamento psicológico, a examinanda poderia melhorar suas condições de como percebe, organiza e dá sentido à sua realidade. Acredita-se que a partir do acompanhamento, a mesma aprenderá a manejar sua ansiedade, enfrentar situações que evita e elaborar o luto das perdas familiares. A psicoterapia é considerada de interesse para que também consiga relatar sobre seu cotidiano e principalmente, para que consiga lidar com as demandas que o meio familiar a impõe, pois a família nuclear e a família extensa influencia diretamente na forma como a paciente pensa e age.

Ademais, considera-se que todos os passos da avaliação psicológica que compõem o psicodiagnóstico foram levantadas. A partir do relato de caso, destaca-se que desde o primeiro atendimento, é tarefa do profissional de Psicologia criar um espaço acolhedor para que o sujeito consiga sentir-se confortável para abordar suas questões. Por fim, aponta-se que ao ser criado este ambiente, o psicólogo deve ficar atento tanto para aspectos enunciados verbalmente quanto aspectos não manifestos no comportamento do examinando.

Acredita-se, que é necessário um maior número de estudos onde foquem na experiência do processo de avaliação psicológica, visto que, a troca de conhecimento sobre o psicodiagnóstico realizado por estudantes de graduação ou psicológos, podem vir a ser de grande auxilio para a formação de outros profissionais. Essa área é de grande importância para a Psicologia, e levando em conta que cada paciente tem sua subjetividade, cada estudo tem caráter único, sendo então, fundamental existirem mais estudos sobre a temática. 


\section{Referências}

Albornoz, A. C. G. (2016). Devolução das informações do psicodiagnóstico. A entrevista de anamnese. O processo psicodiagnóstico. In Hutz, C. S.; Bandeira, D.R.; Trentini, C.; \& Krug, J.S.; Psicodiagnóstico. Porto Alegre: Artmed.

Arpini, D. M., Zanatta, E., Paraboni, P., Rodrigues, P. M. \& Marchesan, R. Q. (2018). Observação e escuta: recursos metodológicos de investigação em psicologia no âmbito da saúde materno-infantil. Contextos Clínicos, 11(2):243-256.

Beck, J. (2013). Terapia Cognitiva-Comportamental: teoria e prática. Artmed: Porto Alegre.

Brasil. Lei $\mathrm{n}^{\circ}$ 4.119, de 27 de agosto de 1962. Dispõe sobre os cursos de formação em Psicologia e regulamenta a profissão de Psicólogo. Disponível em: <http://site.cfp.org.br/wpcontent/uploads/2008/08/lei_1962_4119.pdf>

Britto, I. A. G. S., Oliveira, J. A., \& Sousa, L. F. D. (2003). A relação terapêutica evidenciada através do método de observação direta. Revista Brasileira de Terapia Comportamental e Cognitiva, 5(2),139-149.

Castilho, A. (2002). A dinâmica do trabalho de grupo. Qualitimark. 5(1): 51-92

Coimbra, M. N. C. T. \& Martins, A. M. O. (2013). O estudo de caso como Abordagem metodológica no ensino superior. Nuances. São Paulo, 24(3), 31-46

Conselho Federal de Psicologia (CFP). (2013). Cartilha avaliação psicológica. Brasília: CFP.

Cordioli, A. V. (1998). Psicoterapias: abordagens atuais. Porto Alegre: Artes Médicas.

Cunha, J. A. (2000). Psicodiagnóstico-V. Porto Alegre: Artes Médicas.

Cunha, T. R. S., \& Benetti, S. P. C. (2009). Caracterização da clientela infantil numa clínicaescola de psicologia. Boletim de Psicologia, 59 (130), 117-127. 
Gil, A.C. (2002) Métodos e técnicas de pesquisa social. São Paulo: Atlas.

Guzzo, R. S. L. \& Pasquali, L. (2001). Laudo psicológico: a expressão da competência profissional. In Pasquali, L. (Org). Técnicas de exame psicológico - TEP. Manual: Vol. 1. Fundamentos das técnicas psicológicas. São Paulo: Casa do Psicólogo.

Habigzang, L. F., Stroeher, F. H., Hatzenberger, R., Cunha, R. C., Ramos, M. S. \& Koller, S. H. (2009). Grupoterapia cognitivo-comportamental para crianças e adolescentes vítimas de abuso sexual. Revista de Saúde Pública, 43(Supl 1),70-78.

Hutz, C.S, Bandeira, D. R.; Trentini, C.; \& Krug, J.S.; (2016). Psicodiagnóstico. Porto Alegre: Artmed.

Marques, N. (2005). Entrevista de triagem: espaço de acolhimento, escuta e ajuda terapêutica. In Macedo, M. M. K.; \& Carrasco, L. K. (Con)textos de entrevista: olhares diversos sobre a interação humano. Casa do Psicólogo.

Mônego, B.G. (2016). Cuidados técnicos no início do processo psicodiagnóstico In: Hutz, C.S, Bandeira, D. R.; Trentini, C.; \& Krug, J.S.; Psicodiagnóstico. Porto Alegre: Artmed.

Nunes, C.H.S., Hutz, C.S \& Nunes, M.F.O. (2017). Bateria Fatorial de Personalidade (BFP) - Manual técnico. São Paulo: Pearson Clinical Brasil.

Rigoni, M. \& Sá, S. D. (2016). O processo psicodiagnóstico. In: Hutz, C.S, Bandeira, D. R.; Trentini, C.; \& Krug, J.S.; Psicodiagnóstico. Porto Alegre: Artmed.

Silveira, M. A. \& Bandeira, D. R.; (2016). A entrevista de anamnese. O processo psicodiagnóstico. In Hutz, C.S., Bandeira, D.R., Trentini, C. \& Krug, J.S. Psicodiagnóstico. Porto Alegre: Artmed. 
Trentini, C. M., Hutz, C. S., Bandeira, D. R., Teixeira, M. A. P., Gonçalves, M. T. A., \& Thomazoni, A. R. (2009). Correlações entre a EFN - escala fatorial de neuroticismo e o IFP inventário fatorial de personalidade. Avaliação Psicológica, 8 (2), 209-217.

\section{Porcentagem de contribuição de cada autor no manuscrito}

Gabriela Maffini- 70\%

Paula Argemi Cassel- 30\% 